\title{
Effects of nonopsonized Escherichia coli on myeloperoxidase activity in medium used for incubation of leukocytes from patients with gingivitis and periodontitis
}

\author{
Jonas Žekonis, Gediminas Žekonis and Jurgina Sakalauskiene \\ Kaunas Medical Academy, Department of Prosthetic Stomatology (3000 Kaunas, Mickevičiaus 9)
}

(Received 4 March 1996 and accepted 12 February 1997)

\begin{abstract}
An attempt was made to explore the myeloperoxidase (MPO) activity in medium used for incubation of peripheral venous blood (PVB) leukocytes from patients with gingivitis and periodontitis and to compare it with that of periodontally healthy subjects. The study population included 54 gingivitis patients $(G), 52$ periodontitis patients $(P)$ and 52 control subjects $(C)$. All these groups were assessed by clinical, laboratory and statistical methods. The leukocytes were incubated with opsonized zymosan, Escherichia coli ATCC25922, nonopsonized E.coli or Staphylococcus aureus 256. The respective levels of MPO activity in incubation media of PVB leukocytes taken from group $G$ patients were $598.0 \pm 29.2$ conventional units (c. u.), $640.0 \pm 26.3$ c. u., $662.0 \pm 37.6$ c. u. and $750.0 \pm 40.8$ c. $u$. (control incubation medium: $564.0 \pm 25.1$ c. $u$.); those for group $P$ patients were $672.0 \pm 34.3$ c. u., $678.0 \pm 43.1$ c. u., $692.0 \pm$ 47.9 c. $u$. and $762.0 \pm 34.7$ c. u. ( control: $612.0 \pm 35.2$ c. u.); those for group $C$ subjects were $556.0 \pm 30.2$ c. u., $714.0 \pm 28.2$ c. u., $1276.0 \pm 69.0$ c. u. and $794.0 \pm$ 47.1 c. u. (control: $534.0 \pm 29.0$ c. u.). MPO activity was increased most significantly when nonopsonized E.coli was added to the incubation medium of PVB leukocytes taken from subjects with intact periodontium. MPO activity was unchanged when the leukocytes were taken from periodontitis patients.
\end{abstract}

Key words: periodontal disease; neutrophils; enzymology; myeloperoxidase.

\section{Introduction}

Periodontal disease is considered to be inflammatory disorder whose patho-physiology is related to accumulated dental microbial plaque and the host response to this accumulation ${ }^{[1,2]}$. The host reaction to gingival microorganisms is characterized in part by an influx of polymorphonuclear leukocytes (PMN), which is one of the most important steps in host defense ${ }^{[3]}$. PMN establish the first defense barrier against the microbial challenge ${ }^{[3,4,5]}$. A decreased count of PMN and/or impairment of their function is detrimental to the maintenance of periodontal health ${ }^{[3,6,7,8,9]}$. PMN contain the necessary material for killing pathogenic microorganisms, including MPO, which is considered to play an important role

Correspondence to: Dr. J. Zekonis, Vilniaus $13-28,3000$ Kaunas, Lithuania in the host response, especially in oxygen-mediated defense mechanisms ${ }^{[10]}$. The MPO- $\mathrm{H}_{2} \mathrm{O}_{2}$-halide system has been shown to be effective in killing periodontopathic microorganisms ${ }^{[10,11]}$. Interaction between bacteria and neutrophilic leukocytes activates a complex series of immunological and biochemical events, resulting in the uptake and subsequent killing of the microorganisms ${ }^{[12]}$. MPO is central to these interrelated activities in the MPO-hydrogen peroxide-halide system ${ }^{[13]}$. Studies on the relationship between periodontal disease and MPO activity of crevicular PMN have revealed decreased MPO activity in gingival crevicular neutrophils of subjects without inflammatory changes of the gingival crevice: the enzyme activity is not detected in approximately $50 \%$ of crevicular PMN. All PVB neutrophil MPO activity levels were found to be within the normal range, and the MPO activity of gingival neutrophils was also normal in all subjects with inflamed tissue ${ }^{[14]}$.

However, there seems to be little information about how the leukocytes of patients with periodontal disease release MPO into the surrounding environment in response to the presence of microbes.

The aim of the present study was to determine the MPO activity in medium used to incubate PVB leukocytes taken from patients with periodontitis and subjects with no inflammation of the periodontium.

\section{Patient selection}

\section{Materials and Methods}

Our study patients were selected from a large number of individuals with pathology of periodontal tissues who were examined clinically and radiographically and diagnosed as having gingivitis (gingival inflammation, bleeding on probing) or periodontitis (deep periodontal pockets, destruction of periodontal tissues surrounding the affected teeth and advanced vertical bone loss). They underwent periodontal treatment or routine check-ups at the Department of Stomatology, Kaunas Academic Clinic. We chose and included in our study only those patients with very marked signs of gingivitis and periodontitis using Russell's ${ }^{[15]}$ periodontal index (PI), which are given in Table 1 . The study was performed on 158 systemically healthy subjects within an age range of 18 to 50 years: 54 patients ( 21 females and 33 males) with gingivitis (G), 52 patients (23 females and 29 males) with periodontitis (P) and 52 (29 females and 23 males) periodontally healthy controls $(\mathrm{C})$, chosen from among donors, dental students and personnel. 


\section{Collection of blood samples}

Ten milliliters of venous blood was collected from the subjects into a sterile syringe containing heparin (20 units/ml).

\section{Laboratory studies}

The test tubes with blood were positioned at an angle of 45 degrees and kept for $1 \mathrm{~h}$ at $37^{\circ} \mathrm{C}$. Then the supernatant plasma rich in leukocytes was aspirated. The leukocyte count in the plasma was levelled to $1 \times 10^{9}$ cells / 1 , and $1.5 \mathrm{ml}$ of the fluid was taken for each incubation medium. Then to the first specimen $0.1 \mathrm{ml}$ of phosphate buffer was added (control); to the $2 \mathrm{nd}, 0.1 \mathrm{ml}$ of opsonized zymosan; to the $3 \mathrm{rd}, 0.1 \mathrm{ml}$ of opsonized E.coli; to the 4th, $0.1 \mathrm{ml}$ of nonopsonized E.coli; and to the $5 \mathrm{th}, 0.1 \mathrm{ml}$ of nonopsonized S.aureus.

Zymosan was opsonized by the method of Cates ${ }^{[16]}$; its further preparation as well as the opsonization of E.coli and S.aureus were performed by the method of Peterson and Schmeling ${ }^{[17]}$.

The opsonized and nonopsonized E.coli and S.aureus were used at a concentration of $3 \times 10^{6}$ particles $/ \mathrm{ml}$. In the samples of leukocyte incubation media, prepared by the method of Talstad et al. ${ }^{[18]}$, MPO activity was determined spectro-photometrically in conventional units (the conventional unit is activity causing an increase in absorbancy of 0.001 per minute at $20^{\circ} \mathrm{C}$ ) by the method of Klebanoff $\mathrm{f}^{[19]}$ using the modification of Yankovsky et al. ${ }^{[20]}$. The reaction was followed for $10 \mathrm{~min}$ at $460 \mathrm{~nm}$.

\section{Statistical studies}

Differences among the studied data were evaluated by Student's $t$ test.

\section{Results}

Clinical data. The mean age of the group $\mathrm{P}$ patients was $40.5 \pm 2.7$ years; that of the group $G$ patients was $32.9 \pm 3.1$ years, and that of the group C subjects, $32.5 \pm$ 3.4 years. The difference in mean age between the group $\mathrm{P}, \mathrm{G}$ and $\mathrm{C}$ subjects was not significant $(\mathrm{p}>0.05)$. Russell's PI in the group $C$ subjects equalled 0 ; that in the group $\mathrm{G}$ patients was $1.37 \pm 0.13$, and that in the group $\mathrm{P}$ patients was $5.57 \pm 0.36$ (Table 1 ).

\section{Laboratory data}

It is evident from the data presented in Table 2 that there was no significant difference $(p>0.05)$ between any of the examined groups in their blood plasma percentages of phagocytes.

Data on MPO activity in the samples are given in Figure 1. They show that the most distinct rise of MPO activity occurred in the group $C$ subjects' PVB leukocyte incubation media with nonopsonized E.coli: the activity was more than double that in the leukocyte control incubation media and in the incubation media of leukocytes with opsonized zymosan, and more than 1.5 times as high as that in the incubation media of leukocytes with opsonized E.coli and nonopsonized S.aureus. A significant rise $(\mathrm{p} \leq 0.001)$ of MPO activity was also observed in the PVB leukocyte incubation media with opsonized
E.coli and nonopsonized S.aureus, as compared with the MPO activity in the control leukocyte incubation media.

None of the studied groups $(\mathrm{C}, \mathrm{G}$ and $\mathrm{P})$ showed a significant $(p>0.05)$ elevation of MPO activity in the PVB leukocyte incubation media with opsonized zymosan in comparison with the enzyme activity in control leukocyte incubation media.

A significant rise $(\mathrm{p} \leq 0.05)$ in MPO activity was observed in the group G subjects' PVB leukocyte incubation media with opsonized and nonopsonized E.coli, but there was no significant difference in MPO activity $(\mathrm{p}>$ $0.05)$ between the latter media. A somewhat higher MPO activity was noted $(\mathrm{p} \leq 0.01)$ in the group $\mathrm{G}$ subjects' PVB leukocyte incubation media with nonopsonized S.aureus, significantly ( $\mathrm{p} \leq 0.05$ ) exceeding the analogous value in the PVB leukocyte incubation media with opsonized E.coli, but not differing ( $>>0.05$ ) from the MPO activity in the PVB leukocyte incubation media with nonopsonized E.coli. Interestingly, the latter activity was almost twice as low as that for the analogous group C subjects.

A significant rise in MPO activity was detected only in the PVB leukocyte incubation media of group P subjects with nonopsonized S.aureus $(\mathrm{p} \leq 0.01)$, but the activity did not differ significantly $(\mathrm{p}>0.05)$ from that in the analogous leukocyte incubation media from group $\mathrm{C}$ and $\mathrm{G}$ subjects. In contrast, the activity of group $\mathrm{P}$ MPO assessed in the PVB leukocyte incubation media with nonopsonized E.coli was almost twice as low as the activity in the analogous C group PVB leukocyte incubation media.

\section{Discussion}

The difficulties encountered in determining the etiologic agents of destructive periodontal diseases have been discussed at length ${ }^{[21,22]}$. Investigators have been handicapped by the dual problems of technical difficulty and inadequate understanding of the biology of destructive periodontal diseases ${ }^{[23]}$. As has been shown by investigations in recent years ${ }^{[24,25]}$, periodontal disease is initiated by subgingival infection with selective Gramnegative bacteria, but the presence of microorganisms alone is not the only factor responsible for periodontal destruction $^{[3,26,27]}$. The responses of the host to pathogenic bacteria are thought to be critically important ${ }^{[28]}$.

The determined clinical parameters (Table 1) showed significantly increased PI in both groups ( $\mathrm{G}$ and $\mathrm{P}$ ) of patients in comparison with the group $\mathrm{C}$ subjects. Bearing in mind that interactions between bacteria and the immune system play a central role in the etiology of periodontal disease $\mathrm{e}^{[29,30]}$, it was expected that our selection of subjects with severe periodontal lesions of inflammatory origin in the $\mathrm{G}$ and $\mathrm{P}$ groups would provide clearer data on the interaction between leukocytes and bacteria and their toxins, as compared with the analogous data for the group $\mathrm{C}$ subjects. It is also evident from the data that the mean age of the subjects in the three groups $(\mathrm{G}, \mathrm{P}$ and $\mathrm{C}$ ), and the leukocyte counts in PVB and the incubation media (Table 2) did not differ significantly $(\mathrm{p}>0.05)$. 
Table 1 : Data for study subjects and clinical evaluation

\begin{tabular}{|c|c|c|}
\hline $\begin{array}{c}\text { GROUPS OF } \\
\text { STUDIED SUBJECTS }\end{array}$ & AGE (YR) & $\begin{array}{c}\text { RUSSELL'S } \\
\text { PI }\end{array}$ \\
\hline $\mathrm{C}$ & $29.6 \pm 4.9$ & 0.00 \\
\hline $\mathrm{G}$ & $32.9 \pm 3.9$ & $1.37 \pm 0.13$ \\
\hline $\mathrm{P}$ & $40.5 \pm 3.1$ & $5.57 \pm 0.36$ \\
\hline
\end{tabular}

Table 2 : Leukocyte count and percentage distribution in incubation media

\begin{tabular}{|c|c|c|c|c|}
\hline \multirow{3}{*}{ GROUPS } & \multicolumn{3}{|c|}{ LEUKOCYTES IN INCUBATION MEDIA } \\
\cline { 2 - 5 } & $\begin{array}{c}\text { LEUKOCYTE COUNT } \\
\left(1 \times 10^{9}\right)\end{array}$ & \multicolumn{2}{|c|}{ DIFFERENTIAL LEUKOCYTE COUNT } \\
\cline { 2 - 5 } $\mathbf{C}$ & $10.0 \pm 0.14$ & GRANULOCYTES & $54.5 \pm 7.5$ & $2.7 \pm 1.1$ \\
\hline $\mathbf{G}$ & $10.0 \pm 0.15$ & $42.8 \pm 6.5$ & $50.9 \pm 8.1$ & $2.6 \pm 1.3$ \\
\hline $\mathbf{P}$ & $10.0 \pm 0.12$ & $46.5 \pm 7.6$ & $46.7 \pm 8.6$ & $2.8 \pm 1.6$ \\
\hline $\mathbf{p}$ & & $50.5 \pm 8.1$ & & LYMPHOCYTES \\
\hline
\end{tabular}
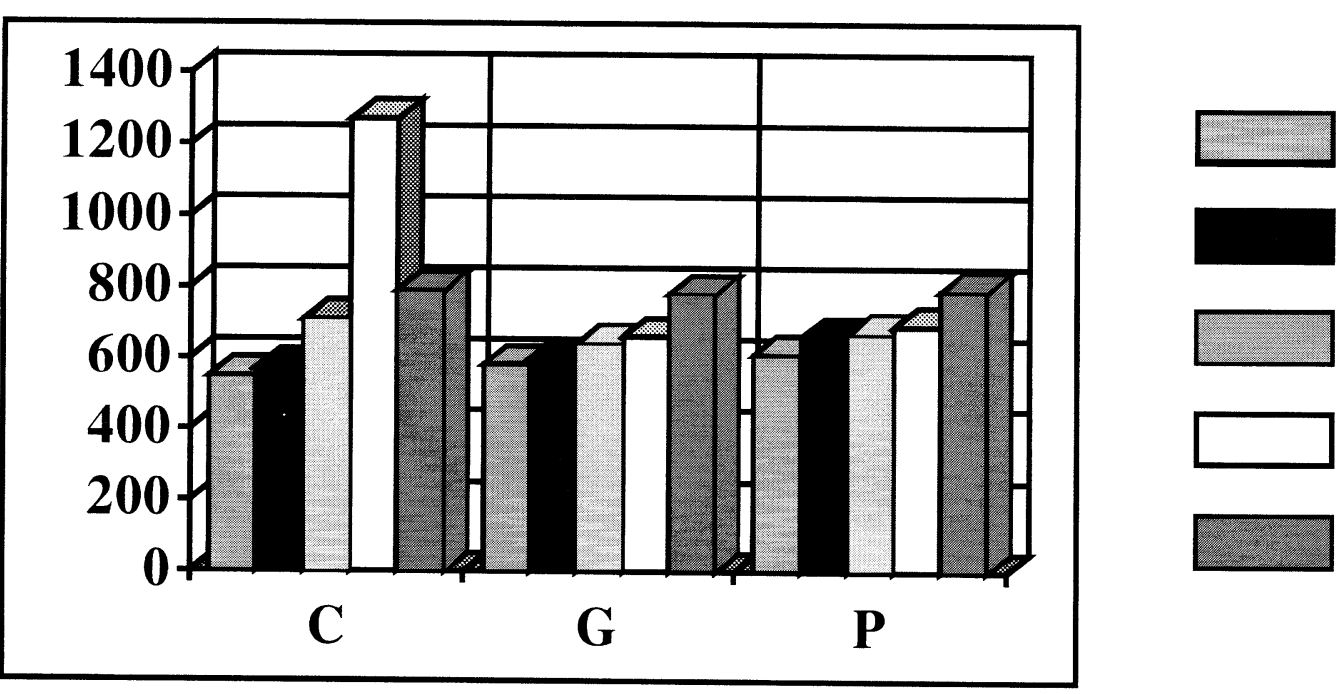

Phosphate buffer

Zymosan (opsonized)

E.coil (opsonized)

E.coil (nonopsonized)

S.aureus (nonopsonized)

Fig. 1 : MPO activity in leukocyte inclubation media (in conventional units)

C, MPO activity in incubation media of leukocytes taken from control group subjects;

G, MPO activity in incubation media of leukocytes taken from patients with gingivitis;

$\mathrm{P}, \mathrm{MPO}$ activity in incubation media of leukocytes taken from patients with periodontitis. 
Hence, the leukocyte count in the incubation media and the mean age of the subjects might not have had any substantial influence on the results for groups $G$ and $P$.

Neutrophils are the principal cells of the host defense system $^{[31]}$ and the primary protective cells against periodontal disease ${ }^{[32]}$. Phagocytosis and the release of lysosomal factors are essential for the bactericidal activity of human polymorphonuclear leukocytes ${ }^{[33]}$. According to Wilton $^{[34]}$, extracellular release of lysosomal factors by neutrophilic leukocytes from gingival crevicular exudate is possibly more efficient than phagocytosis.

MPO is one of the enzymes considered to play a role in the host response, especially oxygen-mediated defense mechanisms $^{[14,35]}$. From this view-point, the results of our study are of special interest: we revealed a very striking increase of MPO activity $(\mathrm{p} \leq 0.001)$ in the group $\mathrm{C}$ PVB leukocyte incubation media with nonopsonized E.coli, being almost double that in the analogous media for groups $\mathrm{G}$ and $\mathrm{P}$. This may indicate that the leukocytes in groups $\mathrm{G}$ and $\mathrm{P}$ did not release enough MPO into their surroundings, and therefore periodontal tissue protection was insufficient. Our findings confirm indirectly the data obtained by Kowolik and Grant ${ }^{[14]}$ indicating that gingival neutrophil MPO levels were normal in all subjects with inflamed tissue, whereas no enzyme activity was detected in approximately $50 \%$ of those with no gingival inflammation. It appeared that some of the MPO had been released by gingival neutrophils of these subjects into the surroundings in response to oral bacteria.

The $\mathrm{C}$ group subjects' MPO activity in incubation media of leukocytes with opsonized E.coli, although significantly ( $\mathrm{p} \leq 0.001$ ) higher than the activity in the control leukocyte media, was, however, significantly lower ( $\mathrm{p} \leq$ 0.001) than that in the PVB leukocyte incubation media with nonopsonized E.coli.

The G group subjects' MPO activity in media used for incubation of leukocytes with opsonized and nonopsonized E.coli was significantly higher ( $\mathrm{p} \leq 0.05$ ) than that in the PVB leukocyte control incubation media, showing no significant difference in the MPO activity ( $\mathrm{p}$ $>0.05$ ) between both of the media with E.coli. In contrast, the group P MPO activity in the leukocyte incubation media with opsonized and nonopsonized E.coli was not essentially different $(\mathrm{p}>0.05)$ from that in the analogous PVB leukocyte control incubation media.

The $\mathrm{C}, \mathrm{G}$ and $\mathrm{P}$ group MPO activity in the leukocyte incubation media with $\mathrm{S}$.aureus was significantly higher than that in the analogous control leukocyte incubation media ( $\mathrm{p} \leq 0.001, \mathrm{p} \leq 0.001$, and $\mathrm{p} \leq 0.01$, respectively), without any significant difference $(\mathrm{p}>0.05)$, but it was significantly $(\mathrm{p} \leq 0.001)$ lower than the activity of group $\mathrm{C}$ MPO in the leukocyte incubation media with nonopsonized E.coli. This indicates that MPO is released in various quantities by the leukocytes in response to different microbes. Various activities of MPO, especially of groups $\mathrm{C}$ and $\mathrm{P}$, of leukocyte incubation media with nonopsonized E.coli, may give some idea of the disturbance of the bactericidal function of leukocytes by individual microbes in subjects with periodontal inflammatory pathology.
The present data confirm the importance of MPO for the normal function of periodontal tissues, although they are at variance with Över et al. ${ }^{[36]}$, who suggested that MPO activity may be related to the pattern and severity of periodontal breakdown.

Further studies are required for a final solution of this problem.

\section{Conclusions}

1. The activity of MPO increases when bacteria, especially nonopsonized bacteria, are added to human leukocyte incubation media.

2. The extent of the increase in MPO activity in the incubation media depends on the species of bacteria added.

3. The activity of MPO does not change significantly if nonopsonized E.coli is added to the incubation media of PVB leukocytes taken from the patients with periodontitis. On the other hand, its activity doubles in the analogous incubation media if the leukocytes are taken from subjects with an intact periodontium.

\section{References}

[ 1 ] Palcanis, K.G., Larjawa, I.K., Wells, B.R., Suggs, K.A., Landis, J.R., Chadwick, D.E. and Jeffcoat, M.K.: Elastase as an indicator of periodontal disease progression. J. Periodontol., 63, 237-242, 1992

[ 2 ] Lamster, I.B., Celenti, R. and Ebersole, J.L.: The relationship of serum IgG anti-body titers to periodontal pathogens to indicators of host respose in crevicular fluid. J. Clin. Periodontol., 17, 421-425, 1990

[ 3 ] Seymour, G.J.: Importance to the host response in the periodontium. J. Clin. Periodontol., 18, 421426, 1991

[ 4 ] Page, R.C. and Schroeder, H.E.: Current status of the host response in chronic marginal periodontitis. J. Periodontol., 52, 477-491, 1981

[ 5 ] Seymour, G.J., Whyte, G.J. and Powell, R.N.: Chemiluminescence in the assessment of the polymorphonuclear leukocyte function in chronic inflammatory periodontal disease. J. Oral Pathol., 15, 125-131, 1986

[ 6 ] Zucchelli, G., Parente, R. and Melotti, C.: Functional PMN defects in young periodontal patients. $J$. Dent. Cadmos, 59, 48-56, 1991

[ 7 ] Cutler, C.W., Eke, P., Arnold, R.R. and Van Dyke, T.E.: Defective neutrophil function in an insulin-dependent diabetes mellitus patients. A case report. $J$. Periodontol., 62, 394-401, 1991

[ 8 ] Izumi, Y., Sugiyama, S., Shinozuka, O., Yamazaki, I., Ohyama, I. and Ishikawa, I.: Defective neutrophil chemotaxis in Down's syndrome patients and its relationship to periodontal destruction. J. Periodontol., 60, 238-242, 1989

[ 9 ] Cohen, D.V. and Morris, A.L.: Periodontal manifestation of cyclic neutropenia. J. Periodontol., 32, 159-168, 1961 
[10] Klebanoff, S.J.: Myeloperoxidase- halide- hydrogen peroxidase antibacterial system. J. Bacteriol., 95, 2131-2138, 1968

[11] Miyasaki, T.K., Wilson, M.E. and Genco, R.J.: Killing of Actinobacillus actinomyce-temcomitans by the human neutrophil myeloperoxidase-hydrogenperoxide-chloride system. Infect. Immun., 53, 161165, 1986

[12] Baehner, R.L. and Boxer L.A.: Morphological and biochemical alterations of poly-morphonuclear neutrophil (PMN) leukocytes from patients with inborn errors of phagocytic function: a comprehensive review. In Inborn Errors of Immunity and Phagocytosis. 201-218, Guttler, F., Seakins, J.W.T., Harkness, R.R., eds., MTP Press, London, UK, 1979

[13] Klebanoff, S.J.: A peroxidase-mediated antimicrobial system in leukocytes. J. Clin. Invest., 46, 1078, 1967

[14] Kowolik, M.J. and Grant, M.: Myeloperoxidase activity in human gingival crevicular neutrophils. Arch. Oral Biol., 2, 293-295, 1983

[15] Russell, A.: A system of classification and scoring for prevalence surveys of periodontal disease. $J$. Dent. Res., 35, 350-359, 1956

[16] Cates, K.: Leukocyte chemotaxis: micropore filter assay. In Investigation of Phagocytes in Disease. 29-35, Douglas, S.D., Quie, P.G., Medicina, Moscow, 1983 (in Russian)

[17] Peterson, P.K. and Schmeling, D.: Opsonization of bacteria and phagocytosis. In Investigation of $\mathrm{Ph}$ agocytes in Disease. 59-72, Douglas, S.D., Quie, P.G. Medicina, Moscow, 1983 (in Russian)

[18] Talstad, I., Dalen, H. and Lehmann, V.: Degranulation and enzyme release during phagocytosis of inert particles and of bacteria by polymorphonuclear neutrophil granulocytes. Acta Pathol. Microbiol. Immunol. Scand. Sect. C., 91, 403-411, 1983

[19] Klebanoff, S.J.: Inactivation of estrogen by rat uterine preparations. Endocrinology, 76, 301-311, 1965

[20] Yankovskij, O.Y. and Ramenskaja, M.P., Kokrekov, V.N. : Metod vydelenija mieloperoksidazy leikocitov krolika i korovy. Vestn. Leningr. Univ., 3, 99-103, 1978 (in Russian)

[21] Socransky, S.S., Haffajee, A.D., Smith, S.L.F. and Dzink, J.L.: Difficulties encountered in the research for the etiologic agents of destructive periodontal diseases. J. Clin. Periodontol., 14, 588-593, 1987

[22] Socransky, S.S. and Haffajee, A.D.: Microbiological risk factors for destructive periodontal diseases. In Risk Assessment in Dentistry. 79-90, Bader, J.D., Chiepel Hill, University of North Carolina Dental Ecology, 1990
[23] Socransky, S.S. and Haffajee, A.D.: The bacterial etiology of destructive periodontal disease: Current Concepts. J. Periodontol., 63 (Suppl. 4), 322-331, 1992

[24] Shelburne, C.E., Sandberg, G.P., Binsfeld, C.A., Wolff, L.F. and Curry, R.A.: Monoclonal antibodies to lipopolysaccharide of four oral bacteria associated with periodontal disease. J. Periodontal Res., 28, 1-9, 1993

[25] Williams, R.C.: Periodontal disease. N. Engl. J. Med., 322, 373-382, 1990

[26] Ranney, R.R.: Immunologic mechanisms of pathogenesis in periodontal diseases: an assessment. $J$. Periodontal Res., 26, 243-254, 1991

[27] Shapira, L., Gordon, B., Warbington, M. and Van Dyke, T.E.: Priming effect of Porphyromonas gingivalis lipopolysaccharide on superoxide production by neutrophils from healthy and rapidly progressive periodontitis subjects. J. Periodontol., 65, 129-133, 1994

[28] Leone, C.W., Clark, W.B. and McArthur, W.P.: Host responses in the etiology and pathogenesis of periodontal disease. Curr. Opin. Dent., 1, 29-36, 1991

[29] Flemmig, T. F.: Immunologische Aspecte Marginaler Parodontopathien. Ausblick Diagnostik und Therapie. Parodontol., 1, 203-222, 1990

[30] Caton, J.G. and Quinonn, C.R.: Etiology of periodontal diseases. Curr. Opin. Dent., 1, 17-28, 1991

[31] Niederman, R., Brunkhorst, B., Smith, S., Weinreb, R.N. and Ryder, M.I.: Ammonia as a potential mediator of adult human periodontal infection: inhibition of neutrophil function. Arch. Oral Biol., 35 (Suppl.), 205S-209S, 1990

[32] Yonemura, T.: Phagocytosis, intracellular killing and interleukin 1 production of polymorphonuclear leukocytes in human periodontal diseases. $J$. Periodontol., 31, 403-423, 1989

[33] Quie, P.G.: Bactericidal function of human polymorphonuclear leukocytes. Pediatrics, 50 , 264270,1972

[34] Wilton, J.M.A.: The role of the polymorphonuclear leukocyte in the control of subgingival plaque formation. J. Periodontal Res., 17, 506-508, 1982

[35] Marquez, L.A. and Dunford, B.: Reaction of compound 3 of myeloperoxidase with ascorbic acid. $J$. Biol. Chem., 215 , 6074-6078, 1989

[36] Over, C., Yamalik, N., Yavuzyilmaz, E., Ersoy, F. and Eratalay, K.: Myeloperoxidase activity in peripheral blood, neutrophil crevicular fluid and whole saliva of patients with periodontal disease. J. Nihon Univ. Sch. Dent., 35, 235-240, 1993 